insidious and gradual as the morbid process itself. In many cases we can only suspect the existence of the disease. It may be months or even years before such suspicions prove to be well founded or set at rest.

Prognosis.-Interstitial nephritis may go on for many years without causing the patient or his friends to think there is much amiss. The typical granular kidney must require a very long time to arrive at its condition. It is even possible that in an adult it may date from an attack of acute interstitial nephritis occurring in infancy. Yet the duration of the symptoms of which complaint is made rarely exceeds a few years, and may be only a matter of months or even weeks. In one of the fatal cases collected symptoms had lasted three weeks. In two the duration was three months, and in three symptoms bad been observed for two, three, and five years respectively. As I have endeavoured to show, the effects of cardio-vascular hypertrophy are to avert, or at all events to compensate, for those of renal disease. Dropsy is reduced or is prevented by the circulatory changes, and uræmia by the polyuria which occur. Cardio-vascular compensation attains its height in interstitial nephritis, and the onset of symptoms depends to a great extent (1) on the failure of such compensation, and (2) on its being carried to excess. In adults failure and dilatation of the heart and degeneration of the bloodvessels are often the chief causes of the fatal symptoms. But in children and also in adults they may be actually due to excess of cardio-vascular compensation. Thus may be produced the violent headaches for which advice is often first sought. Extremely high general tension due to constriction of arterioles, combined with a powerfully acting heart, may cause hæmorrhage or cerebral apoplexy. Constriction of pulmonary arterioles may give rise to dyspncea, asthma, or pulmonary apoplexy, or in the extremities it may cause Raynaud's disease or gangrene, whilst constriction of renal arterioles when carried to excess may, instead of increasing the amount of urine, actually reduce it, and so bring about uræmia as well as further damage to the kidneys. So the prognosis will largely depend on the degree at which cardio-vascular hypertrophy is maintained and on our ability to aid or thwart its progress as required. Bad signs will be commencing heart failure, headaches, vomiting, dyspncea, with diminution of urine, increasing albuminuria, progressive wasting, and pigmentation. It is needless to point out that the prognosis will be more grave when symptoms of Addison's disease are superadded to those of interstitial nephritis. The surroundings of the patient and his liability therefrom to chills and consequent inflammations so often fatal in this disease must be considered in the prognosis. Finally, although the conditions of heart, pulse, skin, and urine make the existence of the disease evident, life may not be endangered unless signs of failing or of excessive cardio-vascular compensation arise. In the first case the child is no better off than the adultboth are doomed; but in the second case treatment may ward off danger for the time.

Treatment.-The treatment is symptomatic rather than eurative; yet it is rational and follows the lines of pathology laid down. In parenchymatous nephritis, when dropsy threatens or is present and cardio-vascular hypertrophy is slow to occur and at first insufficient, we give digitalis, squill, and citrate of caffein, which are not only powerful heart tonios, but also have a constricting effect on the arterioles and thus promote diuresis. The utility of ergot and cantharides may be similarly explained. Citrate and acetate of potash become converted into alkaline carbonates in the "primæ vix," and Brunton, Cash, and Gaskell ${ }^{10}$ have shown that the effect of alkalies is to contract bloodvessels, whilst that of acids is the reverse. Bi-tartrate of potash is also a remote antacid. If it be true, as I have contended, that dropsy is averted or cured by constriction of arterioles and increased cardiac force, we can understand that the use of such drugs is rational. In interstitial nephritis, on the other hand, cardio-vascular compensatory hypertrophy is carried out to perfection, and this, together with consequent polyuria, prevents dropsy and also uræmia from occurring. Hence these drugs-cardio-vascular tonics-are not called for in interstitial nephritis, unless cardio-vascular compensation fails. But it must be admitted that nature's endeavours to cure are at best imperfect. Cardio-vascular hypertrophy may be carried to excess in interstitial nephritis. Thus may be produced headaches or even apoplexy by rupture of vessels owing to excessive tension, dyspncea and possibly asthma

10 Brunton: Disorders of Digestion, 1886 edition, p. 343. by constriction of the pulmonary arterioles, Raynaud's disease and gangrene by constriction of the arterioles in the extremities, and, above all, uræmia by suppression of urine due to constriction of the renal arterioles. The treatment commonly followed in such cases is rational. We lower arterial tension and relieve the beart by giving vascular depressants such as nitro-glycerine, nitrite of amyl, nitrites and nitrates generally; we promote elimination from the skin by warm or vapour baths, liquor ammoniæ acetatis and other diaphoretics, and from the bowels by bydragogue catbartics. Similar treatment is followed in cases of parenchymatous nephritis where pulse tension is high and the heart threatens to give way owing to the obstruction in front. This is the "cardio-vascular depressant" as opposed to the "cardio-vascular excitant" mode of treatment. It is logically combined with catharsis and diaphoresis. So the treatment of interstitial nephritis in some cases consists in counteracting the effects of excessive cardio vascular hypertrophy and at the same time promoting the action of the skin and bowels in order to compensate for deficient elimination through the kidneys. In the later stages of interstitial nephritis, however, the whole system of cardiovascular compensation tends to fall through, and then we must have recourse to the cardio-vascular excitants which are so useful in the early stages of parenchymatous nephritis. Possibly when supra-renal inadequacy is indicated by the presence of pigmentation, treatment by supra-renal extract may be of use, and this extract has also an extreme power of constricting vessels, which therefore may be beneficial in another way. The general conduct of the case aims at prevention of complications arising from chill by supplying suitable clothing and, if possible, climate. As to other complications: iron is always necessary to combat progressive anæmia; dyspepsia due to gastric congestion and irritability is best treated by bismuth, arsenic, and prussic acid; eclampsia may be checked by inhalation of chloroform or by rectal injection of chloral and bromides. Headaches may also be relieved by the latter taken internally, but free purgation is the most rational mode of treatment. Venesection is, I believe, both useless and dangerous in the late eclampsia of interstitial nephritis in children. Catharsis and diaphoresis are the best methods of depletion. Drycupping, however, may be useful in pulmonary odema, pleurisy, and pericarditis. Finally, no drug can cure a damaged kidney. Sooner or later the morbid process going on must end in death. Treatment at best can only be palliative, yet by it we may hope to stave off the evil day, and I am convinced that the guiding principle should be- "Watch the heart and pulse."

Upper Berkeley-street, w.

\section{A REVIEW OF THE OPERATION FOR EXCISION OF THE KNEE, TOGETHER WITH AN ACCOUNT OF A NEW PROCEDURE-OSTEOPLASTIC IXXISION OF THE} KNEE.

Bx C. B. LOCKWOOD, F.R.C.S. ENG.,

ASSISTANT SURGEON IN ST. BAR'THOLOMEW'S HOSPITAL, SURGEON TO THE GREAT NORTHERA FOSPITAL.

IN this communication I propose to describe an operation for excision of the knee which I have performed on several occasions since 1889. Although the operations are not numerous, yet they are enough to show that the proceeding is quite practicable. Moreover, I hope to prove that it has certain advantages over the usual operation. At the outset it is to be understood that it is not to be done in every case of tuberculous arthritis of the knee. On the contrary each should be treated on its merits, and with regard to the kind and degree of the disease, and to the condition and environments of the patient. Thus, during the past ten years I have treated tuberculous arthritis of the knee by rest and local applications, by drainage, by arthrectomy, by the $1 \mathrm{~A}$
1897.

A paper read before the Medical Society of London on March 8th, 
ordinary excision, and by the operation which I am about to descrike. The last was done upon cases of progressive tuberculous arthritis with pulpy synovial membrane and ulceration of cartilage, but with little caries or displacement of the ends of the bones. Before deciding to operate every effort was made to allay the disease by rest and local applications. But it is important not to delay until sinuses have formed. In the last case a sinus had formed, and the operation was followed by a severe outbreak of septic inflammation. As with most new attempts, errors have been made and failures have occurred. All of these will be clearly pointed out for the guidance of anyone who may be encouraged to imitate my example.

The method of excision which I always see performed, and which is described by all our more recent witers, 2 has certain faults which can be overcome. To these are attribut. able some of the ill results by which it is not infrequently followed. For instance, during the last few years I have had three times to remove wedges of bone to straighten limbs which had become flexed and rotated after the knees had been excised by the ordinary method. That operation is briefly as follows. A flap of skin and subcutaneous tissue is lifted from the front of the knee by a curved incision then the capsule and the internal and external lateral ligaments are divided and the patella is taken away, with division of its ligaments ; next, the crucial ligaments are cut and a slice or slices of the articular ends of the femur and the tibia sawn off; and, in addition, the synovial membrane is taken away. Abroad von Esmarch," von Bergmann, ${ }^{4}$ and others $^{5}$ describe the same operation, as do aliso Ollier ${ }^{6}$ of Lyons and Farabeuf 7 A number of others have been described, but as a rule they only differ from one another in the way in which the joint is opened or in the treatment of the patella. Farabeuf ${ }^{8}$ gives no less than six different incisions, and others might be added. However, no operation seems to enjoy the same repute as that which I have just sketched and called the usual operation.

With regard to the ordinary operation I would remark that the mere skin incision is of small importance, provided it fulfils the first of the principles upon which the operation is founded and therefore permits the whole disease to be removed. Sometimes I have used the so-called anterointernal vertical incision of Langenbeck. ${ }^{9}$ because it seemed to give better access to the supra-patellar pouches and to spare the patella and its ligament. But it was found to have the disadvantage of not thorougbly exposing the posterior angles of the joint, especially that behind the outer condyle. So of late the transverse or semilunar incision has been used. But the indiscriminate removal of the patella is a much more serious matter. It brings about the destruction of the attachments of the quadriceps extensor femoris and is one of the main causes of the flexion to which I have just referred. As Sir Charles Bell has pointed out, a peculiar harmony pertains to the contraction and relaxation of opposing groups of muscles. When one group is weakened or destroyed the other being unopposed distorts the limb. Sometimes the patella may be too diseased to be kept, but even then the aponeurosis over it and at either side of it can be preserved. When the patella is sound it ought to be kept for another reason. In one case I was able to peg its sawn surface to the joints of the tibia and femur, both of which had been sawn to receive it. The ligaments, too, perform important functions. In extension of the knee, as is well known, they are all on the stretch and keep the femur and the tibia in the closest contact and prevent rotation. The crucial ligaments are particularly important. They ob. viously prevent displacement of the tibia backwards and forwaids. Also, in extension, as Bruce Young ${ }^{10}$ has shown, they screw home and lock the tibia and femur. The lateral ligaments assist the crucial ligaments and

2 Jacobson: The Operations in Surgery, third edition, 1897, p. 184 et seq. Treves: A Manual of Operative Surgery, 1891, vol. i., p. 684 Marsh: Diseases of the Joints and Spine, $1895, \mathrm{p}$. 331

The Surgeon's Handbook, 1888, p. 317

(18)

5 Allgemeinen und spec

6 Traité des Résections, 1891, tome iii., p. 206 et seq.

Précis de Manuel Opératoire, p. 775.

8 Loc. cit , Figs. 592 to 595 .

B. Young on the Grooves Separating the Patella from the Nenisco-tibial Surfaces of the Femur, and on Locking of the Knee Menisco-tibial Surfaces of the Femur, and on Locking of the Knee-
joint in Full Extension. Memoirs and Memoranda in Anatomy, by Joint in Full Extension. Memoirs and combine with them to bring about that rigidity and mmobility for which the extended knee is remarkable. Obviously in the ordinary operation all this is quite ignored. The shape of the articular ends of the femur and tibia are accessory to this screwing home and locking. And, in addition, the spine of the tibia, being fitted into the intercondylar notch of the femur, effectually prevents lateral displacement of the tibia when the knee is extended. It is hardly necessary to say that the usual operation of resection of the knee sacrifices all the advantages which may be secured by leaving the patella and its ligament, the lateral ligaments, and the crucial ligaments, or by preserving the interlocking of the articular $\epsilon$ nds. Noreover, it leaves bony surfaces which, besides being unsupported by ligaments, are themselves flat and smooth and easily displaced.

To obviate the disadvantages of the usual operation and to retain the advantages which the arrangements of the ligaments and articular ends afford I have. in suitable cases, performed the following operation. First, the knte being bent at half a right angle, a transverse or slightly curved incision is carried across the centre of the ligamentum patellæ, from one tuberosity of the $f \in m u r$ to the other. The ligamentam patellæ is divided and the joint opened. As I have already said, opening the joint by the vertical incision at the inner side of the patella was tried, but was given up because it made the removal of all the synovial membrane difficult or perhaps impossible. This quite outweighs the advantages of the vertical ir cision. Some surgeon have exposed the interior of the knee by sawing through the patella. But this was not done in any of the cases, because that bone is often tuberculous and because it does not unite with so much certainty as the ligament. Moreover, the divided patella is not adapted for fastening to the tibia and femur. The knee-joint having been freely opened and bent to a right angle the extent of the disease is ascertained. Then the synovial membrane is removed with knife and scissors as carefully as if it were full of malignant disease. Sometimes to get at the upper part of the synovial membrane the ends of the transverse incision have had to be carried opvards. If any synovial membrane be left behind tubercle is likely to grow in it ; also it is apt to inflame and secrete fluid which may accumulate and become septic. In removing the synovial membrane it is convenient to leave the part behind the condyles until the femur has been sawn. As the synovial membrane is taken away the ligaments come into view. Healthy ligaments are to be preserved. Whether it is always right to keep both the crucial ligaments remains to be seen. $I t$ is certainly very hard to remove all the synovial membrone without cutting the anterior. Nevertheless, as I have pointed out, the crucial ligaments help very powerfully to hold the tibia and femur together; the posterior one prevents the tibia falling backwards as the leg lies extended on the bed after the ordinary excision there is a tendency for the tibia to fall backwards. Fortunately the posterior crucial ligament can often be preserved and is of service in preventing that accident. The anterior crucial ligament prevents displacement of the tibia forwards, and is therefore less important; it has hardly anything to do with backward displacement. But it would be better to destroy one or both crucial ligaments rather than leave any diseased synovial membrane behind. This was shown by a case in which my anxiety to preserve the crucial ligaments led me to leave behind some synovial membrane which quite spoilt the operation. The patient was a sailor with tuberculous disease of the knee. The disease in the synovial membrane was extensive with considerable ulceration of the articular cartilages. The ligaments were kept; the wound healed by first intention, and he went in six weeks to a convalescent home with the limb in plaster. He had, of course, strict injunctions not to walk on the leg. A month or so afterwards he came back and complained that his leg hurt him when he walked about on it. It was clear from the swelling that the tuberculous process was still progressing, although the union was wonderfully firm considering what he had been doing. My colleague, Mr. Peyton Beale, in my absence, kindly removed the tuberculous disease, which he told me was quite at the back of the joint, behind, I believe, the crucial ligament. The ultimate result in this case was highly satisfactory.

The internal and the external lateral ligaments, being outside the joint, are much easier to save. In the next stage of the operation, after the femur and the tibia have been sawn, these ligaments are of the greatest use in keeping the bones firmly together. So far the operation 
has hardly differed from many others; but in the next step-namely, the treatment of the bones-it is quite different from any I have been able to find described. First, the articalar surface of the patella is sawn off and, if necessary, its cancellous tissue is gouged. Then the trochlear surface of the femur is sawn off, leaving a flat surface upon which the upper part of the patella fits. The femur itself is next raised vertically by the assistant and the greater part of its articular surfaces taken away by two saw cuts which begin at the convexity of the condyles at the edge of the articular cartilage. These saw cuts are carried obliquely into the intercondylar notch of the femur until they converge, entering the notch at the edge of the articular cartilages and below the attachments of the crucial ligaments (vide Fig. 1). In making these cuts the lower part of the rather extensive attachment of the crucial ligament cannot always be saved, but as the upper attachments spread out a good deal the greater part can be saved. The antero-posterior plane of these cuts should be at right angles to the axis of the femur. By these saw cuts a deep notch is made in the femur, leaving some articular carbilage in the front and back of the condyles. This cartrlage is either torn off or removed with a sharp spoon or gouge. The tibia is treated upon the same principles as the femur. With a narrow saw two oblique cuts are carried upwards from the epiphysial line to converge at the base of the spinous process (Fig. 2). Here, again, the articular cartilages can be removed
FIG. 1.

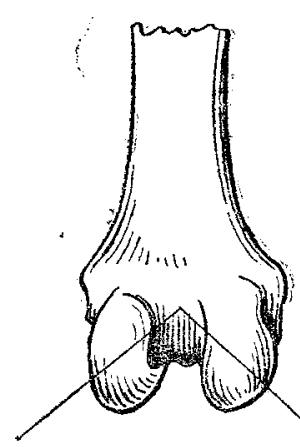

Showing section of femur.
FIG. 2.

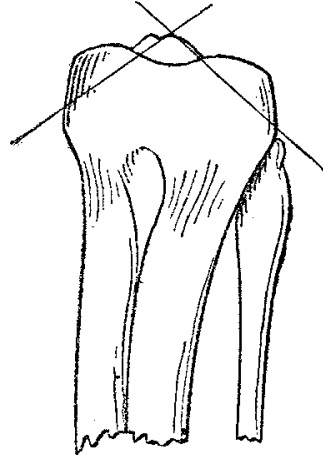

Showing section of tibia. without destroying the lower attachments of the crucial ligaments. This is quite feasible because these structures occupy but a small area of the upper surface of the tibia in front of and behind the spine; indeed, the posterior is attached to the posterior surface of the tibia in the popliteal notch. This stage of the operation is easily accomplished without destroying the lower attachments of either the external or of the internal lateral ligaments, the latter being attached down the shaft of the tibia quite out of harm's way. The antero.posterior direction of the plane of these saw cuts is at right angles to the axis of the tibia; their obliquity should correspond to that of the sawn surfaces of the femur.

The advantages which may be claimed for this method of sawing the femur and tibia are as follows. The original fitting of the tibia into the intercondylar notch is preserved and any lateral displacement of the tibia is still impossible. Further, the same wedging in of the tibia effectually prevents rotation of that bone upon its long axis, and, therefore, the foot falls neither inwards nor outwards. To these advantages may be added that large areas of sawn bone are brought in apposition, and this with the smallest removal of either epiphysis. The whole thickness of the femoral epiphysis is left at either side and the whole thickness of the tibial in the middle. If the knee be extended after the bones have been sawn, the benefit of preserving the ligaments becomes apparent. As the leg is extended they become tight and jam the wedge of the tibia into the notch in the femur. Here it is firmly fixed so long as extension is maintained. As I have pointed out, if the crucial ligaments can be preserved a further advantage is gained, because in extension the posterior prevents the tibia falling backwards and the anterior prevents its displacement forwards. The operation is finished by sewing together the ligamentum patellæ with buried silk sutures and by subsequently closing the wound. In one case the patella was fastened with ivory pegs to the femur and tibia, but lately this has been omitted. The patella seems to form very strong connexions without the pegging. The front of the tibia and the front of the femur were wired together in one case so as to prevent flexion. Afterwards the $\operatorname{limb}$ is kept for a month or six weeks on a Gant's splint. But after this operation the immobility which is so essertial for success is much easier to preserve. Indeed, as the tibia cannot slide to either side or backwards and cannot rotate, we have only to guard against flexicn. That movement is one of the easiest to prevent and has little tendency to occur if the patella has been preserved and its ligament sutured. When the limb comes off tie Gant splint it is put into a leather splint. A patten having been prt on the sound foot the patient goes about on crutches swirging the resected limb. I have hitherto advised that this treatment be continued for a year, but perhaps this time might be shortened.

I have been unable to learn that any surgeon has performed a resection of the knee at all similar to that which has just been described. Mr. Treves ${ }^{11}$ remarks that "Dr. Fenwick of Montreal saws both femur and tibia in a curved line, so as to make them fit together more closely and accurately than they would do otherwise." The bones have been sawn in all kinds of ways ${ }^{12}$ - obliquely by Billroth, in a kind of zigzag by Albert, and the exact reverse of that which I have described by Sédillot. However, none of these methods seem to be based upon the principle of attempting to retain the original fitting of the bones and the action of the ligaments.

The operation which has just been described has been done four times since 1889. A youth who was operated on in 1889 was earning his living three years afterwards and his father wrote: "He gets about freely and does not complain of it in any way ; it is filling cut very nicely and straight." Mr. Mark Taylor, writing about a youth operated upon in April, 1896, says that now "the leg is in very good position; no pain or tenderness anywhere." The photographs which Mr. Taylor sent certainly bear out the statement as to the position of the limb and also show that is is exceedingly well developed. In a third case dove nearly a year ago the patient has a good and straight limb, but I dcubt whether the union is yet bony. She had a septic sinus at the time of operation and the wound suppurated. The fourth case was, as I have already said, a comparative failure, kecause I did not thoroughly remove all the synovial memibrane about the crucial ligaments and behind the condyles. This omission evidently needs to be particularly guarded against.

So far this operation seems to me to have the advantage of treating the bones so as to make it much easier to keep their cut surfaces in contact and at rest; no lateral movement or rotation is possible. Also, under favourable circumstances no falling backwards of the tibia can orcur; next, the growth of the epiphyses is less interfered with; and, finally, the ultimate result is a very solid synostosis, with hardly any tendency to flexion. To gain these advantages the patient runs no risks beyond those of the usual operation.

Upper Berkeley-street, $W$.

\section{A CASE OF ACTINOMYCOSIS TREATED BY IODIDE OF POTASSIUN.}

BY COLVIN B. M. SHITH, M.B.DURH.

IN view of the facts that three cases of actinomycosis, treated by iodide of potassium at the Bristol Royal Infirmary, have been recently recorded ${ }^{1}$ and that there has been some discussion as to the efficacy of the drug as a specific for this disease, the following note of a case may be of interest. A man, a farm-servant, aged twenty-seven years, consulted me on Dec. 7th, 1894, about a swelling in the cheek which caused him pain and prevented him from opening his jaw properly. $\mathrm{He}$ first noticed in October, during the harvest time (in Aberdeenshire) a small lump, "like the head of a pin," on the side of his neck, behind the lobe of the ear. This lump, he said, was moreable and could be pushed up and down under the skin with his finger. This increased in size

\section{Treves : Operative Surgery, vol. i., p. 692.}

12 Allgemeines iber Resectionen, Handbuch der Chirurgie. Pitha und Billroth, 1882, p. 193 